\section{Ornamental creatures}

CHRISTOPHer LEVER's exciting and scholarly book The Naturalized Animals of the British Isles (Hutchinson: London; £7.50) is full of surprises. Did you know that there are Mongolian gerbils on the Isle of Wight, Midwife toads in Bedford, Pumpkinseeds (a species of sunfish) near Tunbridge Wells and Wallabies in Yorkshire? These strange animals and many more are established in the wild in Britain in self-maintaining populations. Many of our more familiar animals have also been introduced by man, including the rabbit, brown rat, house mouse and grey squirrel. Fifty-nine species of vertebrates are described with a separate chapter devoted to each, in which the author describes exactly how, from where and when, the animal was introduced, and summarises its biology and the effects of the introduction on our native flora and fauna.

Many species, such as the pheasant, were introduced on purpose for their food value. Others were brought here for "economic reasons", like the little owl, imported from Italy by an eccentric who had the inspired idea that it would be a useful means of pest control in the kitchen garden and would also rid our church belfries of their sparrows and bats. The difficult question of the advantages and disadvantages of introducing foreign creatures is discussed. Few would deny that we would be better off without the rat; but for Christopher Lever, at least, life in Britain would be miserable without such ornamental creatures as the beautiful and harmless Mandarin duck, to whom he dedicates his fascinating book "with admiration and affection".

Introduced animals, however, can have disastrous effects. Feral dogs on the Galapagos Islands have gone wild and are decimating several of the island populations of land iguanas. A captive breeding project has been set up in an attempt to protect these magnificent beasts. This, together with many other conservation efforts by the World Wildlife Fund (WWF), is described in their yearbook The World of Wildlife edited by Nigel Sitwell (Hamlyn: London; £2.95). The book, which is illustrated with some superb photographs, contains sixteen chapters on endangered species, including the tiger, narwhal, whooping crane and desert pupfish, and describes the efforts that are being made to save them and their habitats. Thanks to the successful Operation Tiger campaign, launched by the WWF, reserves have been set up throughout India, and the tiger now has a good chance of survival.

The most dramatic success of the WWF conservation effort last year must be the Italian government's agreement to give complete protection to the hundred or so remaining wolves in the Apennine mountains. And let us give a thought this Christmas to the beautiful scimitar horned oryx, now in grave danger of extinction. An important breeding centre has been set up in the Marwell Zoological Park in Hampshire, so perhaps we can look forward to the day that the oryx join the wallabies as naturalised British citizens?

I sometimes wonder just why it takes so long for new ideas in the scientific literature to filter through to the popular book market. Inside the Animal World by Maurice and Robert Burton (Macmillan: London; £6.95) is subtitled An Encyclopaedia of Animal Behaviour, and although it provides an extensive catalogue of behaviour, it leaves little room for explanations of how the behaviours could have evolved or what ecological circumstances favour certain behaviour patterns rather than others. Thus, we are told that some species of frogs are good parents though others are not; but the interesting question is why is there a difference?

The vital function of territorial behaviour is said to be the regulation of population numbers so as to prevent overcrowding. But I feel that the layman is entitled to an answer to the question of why the "doomed surplus" accept their miserable role in life. There is next to no reference to the fact that different individuals may be selected during evolution in different ways. How much more fascinating courtship behaviour becomes

\section{Desert habitats}

IN spite of all the constraints desert conditions impose on human activity, they do generate in the individual a great sense of freedom. This, perhaps, explains why so many of those whose names are so closely linked with arid regions were, above all, great individualists. Arabian explorers like Lawrence, Philby and Thesiger have their counterparts in the New World- naturalists who, in their very different way, were no less individualistic and devoted to the desert habitat. Twenty years ago, two books of Edmund C. Jaeger (now in his nineties, and still enjoying the desert environment in Riverside, California) formed an excellent introduction to the North American deserts, particularly those of California. In the early sixties, Alonzo Pond wrote his highly individualistic account of descrts in The Desert World. In the same mould is Raymond B. Cowles. Born and raised in the South African bush, he subsequently spent nearly sixty years studying the adaptation of living organisms to desert conditions in Southern California. He pioneered work on reptilian thermoregulation; and his Desert Journal when we realise that optimal reproductive strategies for males and females are rarely similar. Animals may often signal their sex "because it is a waste of time for a male to court another male", but in some instances it apparently pays a male to mimic female behaviour. Some male elephant seals pretend to be females (the "Danny la Rue strategy") and join a harem to steal copulations, unnoticed by the dominant male. Surely we can no longer be satisfied with explanations of courtship displays as "designed to overcome the fear of close contact", or of lions killing young fathered by other males as a result of a "feeding frenzy". Male lions can in fact enhance their reproductive success by such murderous behaviour, because this brings the females into oestrus again sooner and thus hastens the day that the male can sire his own offspring.

This is, however, an attractive book and will fascinate those readers who want to learn about how wonderful nature is, without wanting to know the reasons why. I feel that the lovely line drawings by Hilary Burn and the outstanding photographs by Jane Burton deserve considerably more acknowledgement than their brief mention on the flyleaf of the dustjacket.

Nicholas Davies

Nicholas Davies is Demonstrator in Zoology at the Edward Grey Institute of Ornithology, Department of Zoology, University of Oxford, $U K$.

(University of California Press: Berkeley, Los Angeles and London. 1977; \$10.95; $£ 8.25$ ) is a reflective account of the background to these studies. The work conveys his enthusiasm, insight and excitement at unravelling the nature of the behavioural and physical adaptations of desert animals to the lethal heat and aridity of their habitat. More than this, it gives the reader an appreciation of the total desert environment.

The Desert, by J. L. CloudsleyThompson (Orbis: London, 1977; £4.95), makes equally easy reading but is quite a different work. Connoisseurs of coffeetable culture may be forgiven if they recall similar offerings from other publishers (Time-Life, Aldus), some with the same title, some with similar photographic credits, some even with the same author. But readers can be assured that the text is different. Also, only in the volume under review are the cold deserts of polar regions considered in addition to hot deserts. At the price, this handsome volume is good value for the layman and widens his choice of introductory books from what must now be an almost saturated market.

M. J. Chadwick

M. J. Chadwick is Senior Lecturer in Biology at the University of York. UK. 